\title{
Last-Train Timetabling under Transfer Demand Uncertainty: Mean-Variance Model and Heuristic Solution
}

\author{
Shuo Yang, ${ }^{1}$ Kai Yang, ${ }^{1}$ Ziyou Gao, ${ }^{1}$ Lixing Yang, ${ }^{1}$ and Jungang Shi ${ }^{2}$ \\ ${ }^{1}$ State Key Laboratory of Rail Traffic Control and Safety, Beijing Jiaotong University, Beijing 100044, China
}

${ }^{2}$ School of Transportation and Logistics, East China Jiaotong University, Nanchang 330013, China

Correspondence should be addressed to Kai Yang; kaiyang@bjtu.edu.cn

Received 12 April 2017; Accepted 28 August 2017; Published 8 October 2017

Academic Editor: Zhi-Chun Li

Copyright (C) 2017 Shuo Yang et al. This is an open access article distributed under the Creative Commons Attribution License, which permits unrestricted use, distribution, and reproduction in any medium, provided the original work is properly cited.

\begin{abstract}
Traditional models of timetable generation for last trains do not account for the fact that decision-maker (DM) often incorporates transfer demand variability within his/her decision-making process. This study aims to develop such a model with particular consideration of the decision-makers' risk preferences in subway systems under uncertainty. First, we formulate an optimization model for last-train timetabling based on mean-variance (MV) theory that explicitly considers two significant factors including the number of successful transfer passengers and the running time of last trains. Then, we add the mean-variance risk measure into the model to generate timetables by adjusting the last trains' departure times and running times for each line. Furthermore, we normalize two heterogeneous terms of the risk measure to provide assistance in getting reasonable results. Due to the complexity of MV model, we design a tabu search (TS) algorithm with specifically designed operators to solve the proposed timetabling problem. Through computational experiments involving the Beijing subway system, we demonstrate the computational efficiency of the proposed MV model and the heuristic approach.
\end{abstract}

\section{Introduction}

The subway is characterized by a high level of safety, wide accessibility, and high energy performance and offers reliable service with sufficient punctuality [1]. Generally, the subway does not provide passengers with 24 -hour service, which means that train operations stop at some point each day (typically late at night or in the very early morning hours) for track or fleet maintenance. The last train naturally becomes the last chance for the passengers transported by the subway to reach their destinations. In addition, the subway usually does not provide a point-to-point transportation service, and the passengers are usually required to transfer one or several times within the network. Statistics show that the last-train transfer passengers accounted for more than $65 \%$ of the total last-train passengers in the entire Beijing subway network in 2015. From the perspective of passengers, they strongly desire well-designed last-train timetables, with good coordination between last feeder trains and last connecting trains so that they can transfer smoothly. Therefore, last-train timetabling should be determined to satisfy the transfer demands.
In many practical situations, the transfer demands of last trains are stochastic. There are several reasons for this, such as inherent stochasticity of real-world traffic flow evolution, multiple sources of data, and fluctuating nature of parameter values. To tackle such type of uncertainty, a samplebased representation may be an appropriate research tool. Based on the historical data, this representation collects the day-specific data, used as stochastic samples with specified probabilities, to capture the characteristics of randomness associated with the transfer demands in subway networks. Furthermore, the scenario-specific samples enable a flexible way for decision-maker (DM) to formulate a model for lasttrain timetabling problem under uncertainty.

The uncertainty of transfer demands can also bring risk to the DM in designing last-train timetables process. The formulation of mean-variance theory is a basic optimization tool to model risk concept with regard to a quadratic utility function for the DM's risk preference. In other words, the DM would want to generate last-train timetables with a higher expected value and a lower variance of positive utilities, such as the number of successful transfers in a risky situation. With 
these concerns, we are particularly interested in developing a systematic approach that synchronously considers stochastic transfer demands characteristics and management of risks in a last-train timetabling problem.

By combining these aforementioned aspects, we intend to address the last-train timetabling problem in uncertain conditions of transfer demands in this paper. For modeling uncertainty, we first employ the mean-variance method to formulate an optimization model for last-train timetabling. Subsequently, we embed the mean-variance risk measure into the model to get timetables for last trains, which is the main novelty of this study compared to previous works. Specifically, this paper aims to make the following contributions to the study of last-train timetable problems.

(i) We adopt the representation of sample-based transfer demands to capture the characteristics of randomness in a subway network. By doing so, the measured transfer demands from real-world applications can be directly embedded in the proposed modeling framework. Furthermore, scenario-specific samples enable a flexible way to measure the uncertainty in decision process, compared to probability function distributions that typically only represent an aggregated version of transfer demands randomness.

(ii) We formulate the mean-variance utility criterion to fully capture the risk preference under uncertainty. With the data structure, we rigorously propose a mean-variance model for last-train timetabling problem that increases the number of successful transfer passengers and at the same time reduces the total running time of last trains. Compared with existing researches, the proposed last-train timetabling framework simultaneously considers uncertainty in the transfer demands and risk modeling.

(iii) We provide a highly efficient method framework to solve the proposed model. In order to eliminate the effect of the heterogeneity between the variance and the expected value in the objective function, we firstly suggest a normalization method by normalizing two heterogeneous terms of the risk measure. Then, we further design a TS algorithm with specifically designed operators to solve the proposed model. The proposed approaches provide a promising algorithmic foundation for solving other closely related last-train timetabling problems in real-life subway networks.

(iv) We conduct a case study based on the Beijing subway instances to demonstrate the effectiveness of the proposed model and solution method. Compared to its counterpart with expected utility criteria, the computational results reported in the numerical experiments demonstrate the superiority of the proposed approach. Further analysis shows that the DM can select distinct values of risk-aversion coefficients depending on their preferences in our optimization method.
The rest of this paper is organized as follows. In Section 2, we briefly review related literature. In Section 3, we give a detailed description for the problem and state the formulation of last-train timetabling problem based on the mean-variance theory. Then, we propose the TS heuristic algorithm to solve the developed model in Section 4. In Section 5, we design a case study based on the real-world operation data of Beijing subway, to demonstrate the effectiveness of the proposed approaches. Finally, some conclusions and further studies are presented in Section 6.

\section{Literature Review}

The literature relevant to this paper comes from two streams: the train timetabling literature and the risk analysis and management literature.

The first stream studies the train timetabling problems. Peeters and Kroon [2] presented a mixed-integer nonlinear programming formulation for cyclic railway timetabling, where the integer variables corresponded to cycles in the graph induced by the constraints. Albrecht and Oettich [3] proposed an algorithm for the dynamic modification of train running times to increase the probability of making connections to other means of public transport. Liebchen [4] formulated a timetable optimization model that optimized the arrival and departure times at the transfer stations of the metro network in Berlin so that the passenger transfer waiting times were minimized. Stanojević et al. [5] developed an integer programming approach for determining an optimal timetable for a single, one-way track linking two major stations. Yin et al. [6] established linear mixed-integer programming models for timetable optimization to decrease the energy consumption and simultaneously to shorten the passengers' waiting time. Gao et al. [7] investigated a rescheduling problem on a metro line in an overcrowded situation after disruptions. Huang et al. [8] studied a joint train scheduling optimization with service quality and energy efficiency in urban rail transit networks, which was solved by a heuristic.

Addressing the last-train timetabling problem in subway networks is a new branch in the train timetabling problem domain. Zhou et al. [9] built coordination optimization models of the last trains' departure time to reduce passengers' transfer waiting time for the last trains and inaccessible passenger volume of all origin-destination (OD) and improve passengers' accessible reliability for the last trains. Kang et al. [10] proposed a rescheduling model for last trains with the consideration of train delays caused by incidents that occurred in train operations. Kang et al. [11] established a lasttrain network transfer model to maximize passenger transfer connection headway, which reflected last-train connections and transfer waiting time. Li et al. [12] studied the last-train coordination problem for metro networks based on actual passenger demand obtained from AFC system data, aiming to maximize the total number of passengers who can reach their destinations by metro prior to the end of operation.

In addition, there is other research concerned with the train timetabling problem under uncertainty. Yang et al. [13] formulated an expected value goal-programming model for 

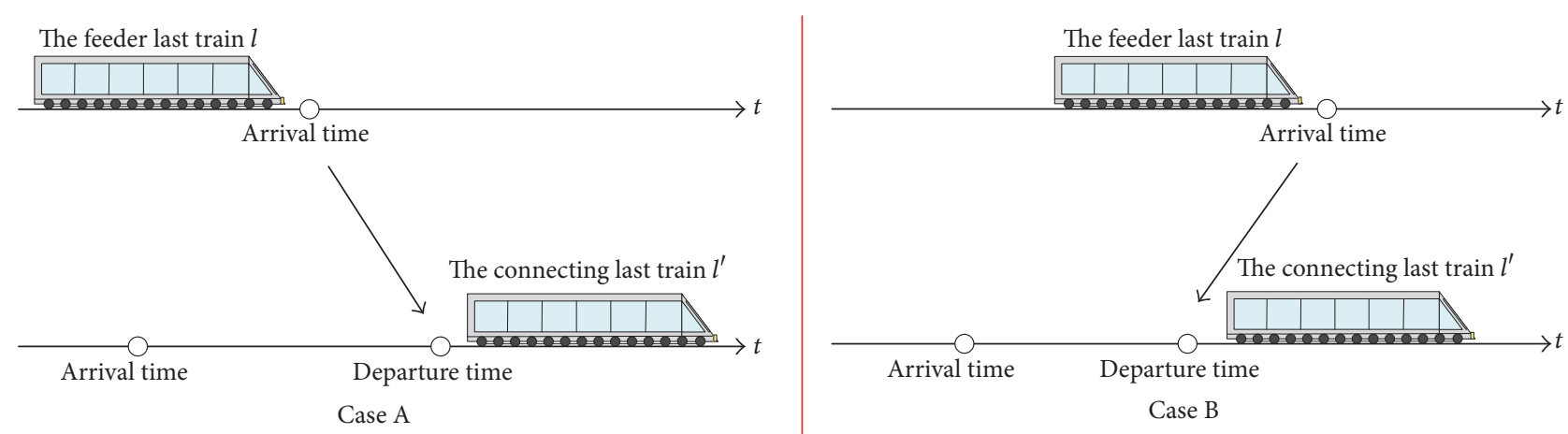

FIGURE 1: Transfer situations based on the arrival and departure times of last trains.

the train timetable problem with fuzzy passenger demand on a single-line railway, in which two objectives, that is, fuzzy total passengers' time and total delay time, were considered. Barrena et al. [14] studied the design and optimization of train timetables for a rail rapid transit line to minimize the average passenger waiting time in a dynamic demand environment, in which an adaptive large neighborhood search metaheuristic was proposed to solve large instances of the problem. Yin et al. [15] developed a stochastic programming model for metro train rescheduling problem in order to jointly reduce the time delay of affected passengers, their total traveling time, and operational costs of trains. Yin et al. [16] converted the train operation problem into a Markov decision process with nondeterministic state transition probabilities to minimize the cost for both the total time delay and energy consumption in a subway line.

The second stream of literature related to this paper is on risk analysis and management. Choi et al. [17] presented a risk assessment methodology for underground construction projects with an uncertainty model based on fuzzy concept. Hensher et al. [18] used a nonlinear probability weighting function to accommodate choice made under risk in the context of the expected utility framework. Woodward et al. [19] designed the flood risk strategies to allow for flexible adaptive measures by capturing the concepts of real options and multiobjective optimization to evaluate potential flood risk management opportunities. Zio [20] examined a framework of vulnerability and risk analysis in relation to its application for the protection and resilience of critical infrastructure. Liu et al. [21] presented a risk analysis of derailment rates on Class I railroad mainlines based on data from the US Federal Railroad Administration and the major freight railroads.

In regard to mean-variance risk measure, Markowitz [22] firstly introduced the mean-variance model into financial portfolio management. Choi et al. [23] used the meanvariance theory to quantify the risk and the payoff of an investment decision by the variance of return and the expected return, respectively. Fu et al. [24] investigated a continuous-time mean-variance portfolio selection problem under the constraint of a borrowing rate which is higher than the lending rate. Wu et al. [25] presented a mean-variance optimization model for solving the track allocation problem that minimizes the occupation time costs in groups of turnouts at station bottlenecks. Ahmadi et al. [26] developed a systematic approach to make robust decisions for the single location-allocation $p$-hub median problem based on mean-variance theory. Li et al. [27] presented a systematic approach integrated the mean-variance and scheduling to allow for schedule delay and trip time variability under uncertainty.

To the best of our knowledge, there has been no significant attempt to study the last-train timetabling problems in stochastic environments considering reliability of decisions; even stochastic models of this problem without considering risk are quiet rare in the literature. This paper takes the first initiative to develop a systematic risk management approach to make decisions according to decision-makers' preferences for a last-train timetabling problem. In this study, more comprehensive and realistic factors are taken into account; these features make this study different from the literature in last-train timetabling area.

\section{Problem Description and Formulations}

Last-train timetabling problem aims at coordinating the arrival and departure times of feeder trains with connecting trains at transfer stations so that passengers can enjoy smooth transfers in subway networks. The practical transfer situations based on the arrival and departure times of the last feeder train and the last connecting train is illustrated in Figure 1. Case A depicts that the passengers can smoothly transfer from train $l$ to train $l^{\prime}$ since the departure time of the train $l^{\prime}$ is later than the passengers' arrival time at the platform of train $l^{\prime}$. Conversely, the transfer passengers cannot do that before the train $l^{\prime}$ departs, as shown in the case B. That is, the passengers fail to transfer in this case. Therefore, lasttrain timetabling should be determined to satisfy the transfer demands. With these concerns, we generate timetables to pick up as many transfers as possible by adjusting the last trains' departure times and running times for each line.

Next, we first present the basic setting upon which our modeling framework is built. We also present several underlying assumptions and most of the notation considered throughout the paper. Then, we propose a modeling framework that synchronously considers uncertainty in the transfer demand and risk modeling, which will be discussed in the following subsections. 
3.1. Basic Model. In this section, we introduce the basic setting for our analysis. We consider a simple base situation in order to get a more focused demonstration of the methodology we propose.

To provide a precise statement of this problem, we define the following:

Sets

$L$ : the set of last trains in the network, $l \in L$.

$S(l)$ : the set of all transfer stations on train $l, s \in S(l)$.

$S_{1}(l, s)$ : the set of transfer stations up to station $s$ on train $l$.

$S_{2}(l, s)$ : the set of transfer stations before station $s$ on train $l$.

\section{Parameters}

$t_{s l l^{\prime}}^{\text {walk }}$ : the passengers' average walking time from train $l$ to $l^{\prime}$ at station $s$.

$d_{s l l^{\prime}}$ : the transfer demand from trains $l$ to $l^{\prime}$ at station $s$.

$t_{s l}^{\mathrm{Dwl}}$ : the dwell time of the last train $l$ at station $s$.

$t_{s l \text { min }}^{R}$ : the minimum running time on train $l$ between station $s$ and the previous transfer station, including additional time caused by train start and brake at stations.

$t_{s l \text { max }}^{R}$ : the maximum running time on train $l$ between station $s$ and the previous transfer station, including additional time caused by train start and brake at stations.

$T_{\min }$ : the earliest permitted departure time for last trains.

$T_{\max }$ : the last permitted departure time for last trains.

\section{Decision Variables}

$t_{o l}^{D}$ : the departure time of the last train $l$ from the starting station.

$t_{s l}^{A}$ : the arrival time of the last train $l$ at transfer station $s$.

$t_{s l}^{D}$ : the departure time of the last train $l$ from transfer station $s$.

$t_{s l}^{R}$ : the segment running time of the last train $l$ between station $s$ and the previous transfer station, including additional time caused by train start and brake at station.

$t_{s l l^{\prime}}^{d}$ : the delay on the platform of $l^{\prime}$ for passengers who transfer from $l$ to $l^{\prime}$ at station $s$.

$x_{\text {sll }}$ : a binary variable which takes a value of 1 if passengers successfully transfer from train $l$ to $l^{\prime}$ at station $s$ and a value of 0 otherwise.

Assumptions 1. To facilitate the presentation of the essential ideas without loss of generality, the following basic assumptions are made in this study:
(A1) The passengers entering the system during the lasttrain period proceed directly to trains and do not linger or engage in nontravel activities (purchasing coffee, etc.).

(A2) The passengers' average walking time between two lines at a station is fixed. With fixed passenger's average walking time, the model can be simplified significantly.

(A3) The last trains' dwelling time at transfer stations is fixed with the same value. The simplicity makes the analysis of processing data more convenient.

(A4) The capacity of each last train is sufficient to receive all passengers. The assumption is reasonable because the passenger volume in last trains is smaller than the train capacity.

Objective Functions. This part analyzes two objectives in the problem of interest, that is, the number of successful transfer passengers and the running time of all the last trains. Besides, we are interested in formulating the objectives into linear formulas that could make the model easier to be handled and also provide a basis for subsequent heuristic solution algorithm.

(i) Total of Running Time. One of the most significant indicators in last-train timetabling is the total of running time for last trains in the view of the DM. The sum of last trains' running time is represented as

$$
F_{1}=\sum_{s \in S(l)} \sum_{l \in L} t_{s l}^{R}
$$

which is introduced to save the operational time and the passengers' traveling time, thus avoiding operational costs and energy wastage.

(ii) Number of Successful Transfer Passengers. The other significant indicator is the number of successful transfer passengers by comparing the arrival time of feeder trains and the departure time of connecting trains. The number of successful transfer passengers can be calculated as

$$
F_{2}=\sum_{s \in S(l)} \sum_{l \in L} \sum_{l^{\prime} \in L} d_{s l l^{\prime}} \cdot x_{s l l^{\prime}},
$$

where $x_{s l l^{\prime}}=0$ indicates that the passengers cannot make the change from $l$ to $l^{\prime}$.

To obtain a tradeoff solution, we give two weight factors, that is, $w_{1}$ and $w_{2}$, to, respectively, indicate the importance of each objective. The last-train timetabling problem can be expressed by the following mathematical program, which we call the basic model:

$$
\begin{array}{ll}
\min & f(T, D)=w_{1} \cdot F_{1}-w_{2} \cdot F_{2} \\
\text { s.t.: } & t_{s l}^{A}=t_{o l}^{D}+\sum_{s^{\prime} \in S_{1}(l, s)} t_{s^{\prime} l}^{R}+\sum_{s^{\prime} \in S_{2}(l, s)} t_{s^{\prime} l}^{D w l}
\end{array}
$$




$$
\begin{aligned}
& t_{s l}^{D}=t_{o l}^{D}+\sum_{s^{\prime} \in S_{1}(l, s)} t_{s^{\prime} l}^{R}+\sum_{s^{\prime} \in S_{1}(l, s)} t_{s^{\prime} l}^{\mathrm{Dwl}} \\
& t_{s l^{\prime}}^{d}=t_{s l^{\prime}}^{D}-t_{s l}^{A}-t_{s l l^{\prime}}^{\mathrm{walk}} \\
& M \cdot\left(x_{s l l^{\prime}}-1\right) \leq t_{s l l^{\prime}}^{d} \leq M \cdot x_{s l l^{\prime}}, \quad x_{s l l^{\prime}} \in\{0,1\} \\
& t_{s l \min }^{R} \leq t_{s l}^{R} \leq t_{s l \max }^{R} \\
& T_{\min } \leq t_{o l}^{D} \leq T_{\max } .
\end{aligned}
$$

Constraints (4) and (5) are operational constraints. $\forall s \in$ $S(l), \forall l \in L$, constraint (4) is given to tracks the arrival time, $t_{s l}^{A}$, of the last train $l$ at station $s$, where $\sum_{s^{\prime} \in S_{1}(l, s)} t_{s^{\prime} l}^{R}$ denotes the total segment running time of the last train $l$ from the starting station to station $s$, and $\sum_{s^{\prime} \in S_{2}(l, s)} t_{s^{\prime} l}^{\mathrm{Dwl}}$ means the total dwell time along the way to station $s$. Constraint (5) states that the departure time of train $l$ at station $s, t_{s l}^{D}$, can be obtained by adding the dwell time $t_{s l}^{\mathrm{Dwl}}$ at station $s$ to $t_{s l}^{\mathrm{A}}$, in which the set $S_{1}(l, s)$ contains transfer stations passed through by the last train $l$ including the current station $s$, and the set $S_{2}(l, s)$ contains members of $S_{1}(l, s)$ except station $s$, since the arrival time at a certain station has nothing to do with how long it will stay here later.

Constraints (6) and (7) are qualitative constraints. In constraint (6), $t_{s l l^{\prime}}^{d}$ represents the difference between the departure time of the connecting train $l^{\prime}$ and the arrival time of transfer passengers from the feeder train $l$ at the platform of train $l^{\prime}$. It is determined by the departure time of the connecting train $l^{\prime}, t_{s l^{\prime}}^{D}$, the arrival time of the feeder train $l, t_{s l}^{A}$, and the passengers' walking time from platform of $l$ to the one of $l^{\prime}, t_{s l l^{\prime}}^{\text {walk }}$. In constraint (7), a binary variable $x_{s l l^{\prime}}$ is introduced to indicate whether passengers can successfully transfer from $l$ to $l^{\prime}$ at station $s$. Here, $M$ is a sufficiently large positive number. That is, $x_{s l l^{\prime}}=1$ if the passengers succeed in transferring from $l$ to $l^{\prime}$ at station $s ; x_{s l l^{\prime}}=0$, otherwise.

Constraints (8) and (9) are time-range constraints. There are upper and lower bounds on segment running time for each interval in the subway network [28], and the departure times of last trains at starting stations are also supposed to be in a limited range for all trains $l \in L$, stations $s \in S$.

\subsection{Mean-Variance Model}

3.2.1. Uncertainty in the Transfer Demands. As it is mentioned in the introductory section, stochastic variability (randomness) always exists in the last-train timetabling problems. In this study, we use the sample-based representation to characterize the randomness associated with the transfer demands. Specifically, we collect a set of $N$ day transfer demands during the last-train period (between 22:00 and 24:00 daily) acquired from the automated fare collection (AFC) system, used as stochastic samples. Each day corresponds to one sample with the even probability $1 / D$. Alternatively, if we use the notation sets $\left\{d_{1}, d_{2}, \ldots, d_{D}\right\}$ to represent the considered days and their probabilities, then the probability of each day-specified data set is deduced by $p_{d_{1}}=p_{d_{2}}=\cdots=p_{d_{D}}$.

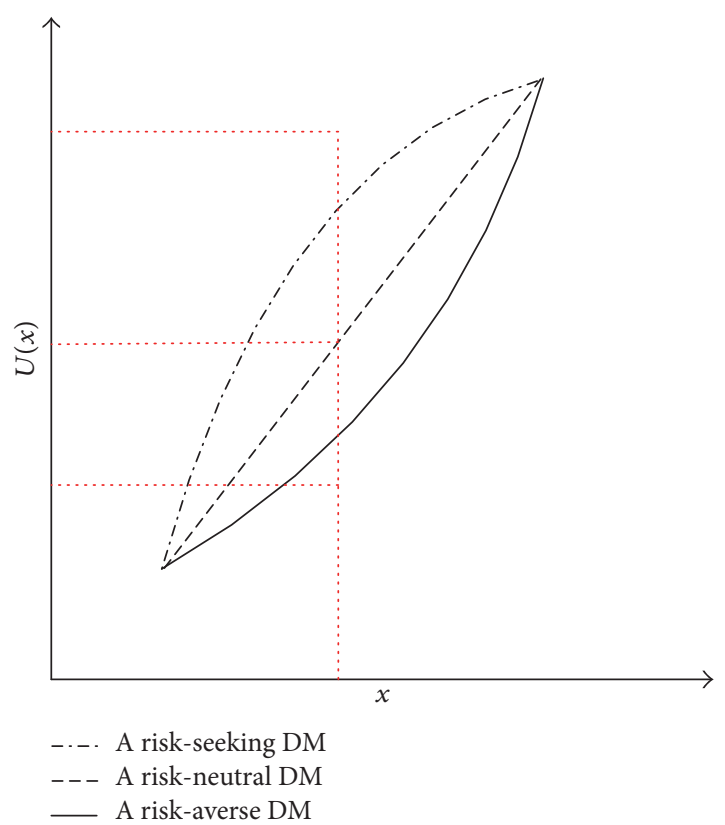

FIgURE 2: The behavior of DM based on quadratic utility function.

Note that, we only consider discrete type of uncertainty where uncertainty is represented by a finite set of samples. When this is not the situation, one can resort to an approximate sampling technique $[29,30]$ and reduce the problem to the finite-scenario setting that we address. Hereafter, we are intended to use the sample-based representation method proposed above to generate the corresponding random variables and then present an optimization model for last-train timetabling problem based on the mean-variance theory.

3.2.2. Mean-Variance Theory. In a risky situation, the DM exhibits three different behaviors in the forms of riskseeking, risk-neutral, and risk-averse that originated from concavity, linearity, and convexity of their utility function [26], respectively, as shown in Figure 2. Apparently, a rational DM prefers to choose a policy with a higher expected value and a lower variance of positive utilities. And in general, the DM has a risk-aversion behavior to deal with a risky situation. Therefore, in this study, we assume that the DM is risk-averse and trying to make decisions with less risk.

The formulation of mean-variance theory is a basic optimization tool to model risk concept with regard to a quadratic utility function for the DM's preference. In this situation, a tradeoff between risk and utility function can be formulated by using a risk-aversion coefficient $\lambda$, given as follows:

$$
U(x)=x+\lambda \cdot x^{2}
$$

in which risk-seeking, risk-neutral, and risk-averse behavior is adjusted based on the negative, zero, and positive value for risk-aversion coefficient $\lambda$, respectively. Therefore, in the stochastic situation with random variable $\xi$, the quadratic 
utility function (positive utility) with a risk-averse preference can be derived as

$$
U(f(x, \xi))=\min \left\{\mu_{f(x, \xi)}+\lambda \cdot \sigma_{f(x, \xi)}^{2} \mid \lambda>0\right\}
$$

where $\mu$ and $\sigma^{2}$ represent the expected value and variance of the function $f(x, \xi)$, respectively.

3.2.3. Mean-Variance Risk Model. Due to the stochastic trait of transfer demands, it is necessary to have an especial tool to analyze the last-train timetabling problem and generate timetables. Moreover, the adopted timetable should be able to meet the subway system requirements for a long time, since changing the implemented initial timetable would be very costly. This clarifies the importance of obtaining solutions with higher reliability or lower risk in the lasttrain timetabling. Based on this consideration, we present a novel approach, considering risk management in the lasttrain timetabling problem under uncertainty. In addition to the parameters introduced in the previous section, we now define the following:

$$
\begin{aligned}
& \xi_{n}: \text { the index of scenarios, } n \in\{1,2, \ldots, N\} . \\
& d_{s l l^{\prime}}^{\xi_{n}}: \text { the number of passengers transferring from } \\
& \text { trains } l \text { to } l^{\prime} \text { at station } s \text { under scenario } \xi_{n} . \\
& p_{\xi_{n}} \text { : the probability of scenario } \xi_{n} .
\end{aligned}
$$

As stated above, we can reformulate the objective function with stochastic transfer demands as follows:

$$
f\left(T, \xi_{n}\right)=w_{1} \cdot A(T)-w_{2} \cdot B\left(T, \xi_{n}\right),
$$

where

$$
\begin{aligned}
A(T) & =\sum_{s \in S(l)} \sum_{l \in L} t_{s l}^{R} \\
B\left(T, \xi_{n}\right) & =\sum_{s \in S(l)} \sum_{l \in L} \sum_{l^{\prime} \in L} d_{s l l^{\prime}}^{\xi_{n}} \cdot x_{s l l^{\prime}} .
\end{aligned}
$$

To obtain the expected value and variance of the above function $f\left(T, \xi_{n}\right)$, the basic terms are introduced as

$$
\begin{aligned}
\mathrm{E}[A(T)] & =A(T), \\
\mathrm{E}\left[B\left(T, \xi_{n}\right)\right] & =\sum_{n=1}^{N} p_{\xi_{n}} \cdot B\left(T, \xi_{n}\right), \\
\mathrm{E}\left[B^{2}\left(T, \xi_{n}\right)\right] & =\sum_{n=1}^{N} p_{\xi_{n}} \cdot B^{2}\left(T, \xi_{n}\right) .
\end{aligned}
$$

By applying the main concept of the first and second moment and substituting (14), the expected value and variance of $f\left(T, \xi_{n}\right)$ are derived as (15) and (16), respectively.

$$
\begin{aligned}
\mathrm{E}[ & \left.f\left(T, \xi_{n}\right)\right]=\mathrm{E}\left[w_{1} \cdot A(T)-w_{2} \cdot B\left(T, \xi_{n}\right)\right]=w_{1} \\
& \cdot A(T)-w_{2} \cdot \mathrm{E}\left[B\left(T, \xi_{n}\right)\right]=w_{1} \cdot \sum_{s \in S(l)} \sum_{l \in L} t_{s l}^{R}-w_{2} \\
& \cdot \sum_{n=1}^{N} p_{\xi_{n}} \cdot B\left(T, \xi_{n}\right), \\
\operatorname{Var} & {\left[f\left(T, \xi_{n}\right)\right]=\mathrm{E}\left[\left(f\left(T, \xi_{n}\right)-\mathrm{E}\left[f\left(T, \xi_{n}\right)\right]\right)^{2}\right] } \\
& =\mathrm{E}\left[\left(w_{1} \cdot A(T)-w_{2} \cdot B\left(T, \xi_{n}\right)-w_{1} \cdot A(T)+w_{2}\right.\right. \\
& \left.\left.\cdot \mathrm{E}\left[B\left(T, \xi_{n}\right)\right]\right)^{2}\right]=\mathrm{E}\left[\left(w_{2} \cdot \mathrm{E}\left[B\left(T, \xi_{n}\right)\right]-w_{2}\right.\right. \\
& \left.\left.\cdot B\left(T, \xi_{n}\right)\right)^{2}\right]=\mathrm{E}\left[w _ { 2 } ^ { 2 } \cdot \left(\left(\mathrm{E}\left[B\left(T, \xi_{n}\right)\right]\right)^{2}\right.\right. \\
& \left.\left.-2 \mathrm{E}\left[B\left(T, \xi_{n}\right)\right] \cdot B\left(T, \xi_{n}\right)+B^{2}\left(T, \xi_{n}\right)\right)\right]=w_{2}^{2} \\
& \cdot\left(\left(\mathrm{E}\left[B\left(T, \xi_{n}\right)\right]\right)^{2}-2\left(\mathrm{E}\left[B\left(T, \xi_{n}\right)\right]\right)^{2}\right. \\
& \left.+\mathrm{E}\left[B^{2}\left(T, \xi_{n}\right)\right]\right)=w_{2}^{2} \cdot\left(\mathrm{E}\left[B^{2}\left(T, \xi_{n}\right)\right]\right. \\
& \left.-\left(\sum_{n=1}^{N} p_{\xi_{n}} \cdot B\left(T, \xi_{n}\right)\right)^{2}\right), \\
& \left.\left(\mathrm{E}\left[B\left(T, \xi_{n}\right)\right]\right)^{2}\right)=w_{2}^{2} \cdot\left(\sum_{n=1}^{N} p_{\xi_{n}} \cdot B^{2}\left(T, \xi_{n}\right)\right. \\
& \\
&
\end{aligned}
$$

where $\mathrm{E}(\cdot)$ and $\operatorname{Var}(\cdot)$ are the expected value and variance mathematical operators.

Therefore, the quadratic utility function based on meanvariance theory could be written as follows:

$$
\begin{aligned}
U\left(f\left(T, \xi_{n}\right)\right)=\mathrm{E}\left[f\left(T, \xi_{n}\right)\right]+\lambda \cdot \operatorname{Var}\left[f\left(T, \xi_{n}\right)\right], & \\
\lambda & >0,
\end{aligned}
$$

where $\lambda$ is the risk-aversion coefficient for the mean-variance function that takes different values depending on the DM's preference in dealing with risky conditions.

The heterogeneity between the variance and the expected value of the function $f\left(T, \xi_{n}\right)$ leads to a difficulty in computing the quadratic utility function. It can be seen that greater values of variance compared to the expected value will lead to loss of sensitivity of the model in different scenarios. In order to reduce this deficiency, the objective function is normalized as suggested in multiobjective optimization approaches. In this research, the two functions of $\mathrm{E}\left[f\left(T, \xi_{n}\right)\right]$ and $\operatorname{Var}\left[f\left(T, \xi_{n}\right)\right]$ are individually optimized in both minimum and maximum states and then the risk measure is defined as the objective function of risk modeling, which can be minimized as follows:

$$
\begin{aligned}
U_{1}\left(f\left(T, \xi_{n}\right)\right) \\
=\frac{\mathrm{E}\left[f\left(T, \xi_{n}\right)\right]-\mathrm{E}_{\min }^{*}\left[f\left(T, \xi_{n}\right)\right]}{\mathrm{E}_{\max }^{*}\left[f\left(T, \xi_{n}\right)\right]-\mathrm{E}_{\min }^{*}\left[f\left(T, \xi_{n}\right)\right]}+\lambda \\
\\
\quad \cdot\left(\frac{\operatorname{Var}\left[f\left(T, \xi_{n}\right)\right]-\operatorname{Var}_{\min }^{*}\left[f\left(T, \xi_{n}\right)\right]}{\operatorname{Var}_{\max }^{*}\left[f\left(T, \xi_{n}\right)\right]-\operatorname{Var}_{\min }^{*}\left[f\left(T, \xi_{n}\right)\right]}\right) .
\end{aligned}
$$




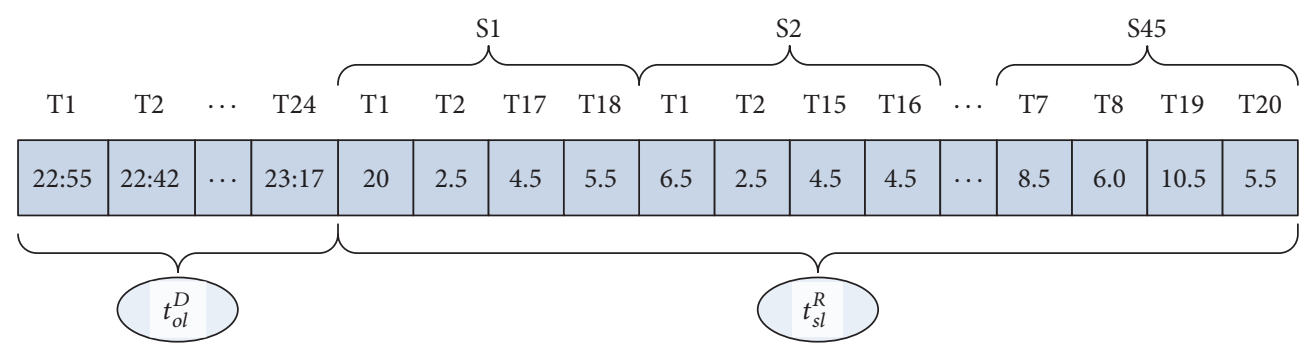

FIGURE 3: Solution representation.

\begin{tabular}{|c|c|c|c|c|c|c|c|c|c|c|c|c|c|c|c|c|c|}
\hline & 1 & 2 & $\cdots$ & 24 & 25 & 26 & 27 & 28 & 29 & 30 & 31 & 32 & $\ldots$ & 203 & 204 & 205 & 206 \\
\hline & $22: 55$ & $22: 42$ & $\cdots$ & 23:17 & 20 & 2.5 & 4.5 & 5.5 & 6.5 & 2.5 & 4.5 & 4.5 & $\ldots$ & 8.5 & 6.0 & 10.5 & 5.5 \\
\hline Select & & Љ & & & Љ & & & & 马 & Љ & & & & & & Љ & \\
\hline Change & & $22: 47$ & & & 23.3 & & & & 5.4 & 2.7 & & & & & & 11.9 & \\
\hline Substitute & $22: 55$ & 22:47 & & $23: 17$ & 23.3 & 2.5 & 4.5 & 5.5 & 5.4 & 2.7 & 4.5 & 4.5 & $\ldots$ & 8.5 & 6.0 & 11.9 & 5.5 \\
\hline
\end{tabular}

FIgURE 4: Neighborhood structure.

On the basis of aforementioned analysis, we can formally present the corresponding mean-variance (MV) model for last-train timetabling problem:

$$
\begin{aligned}
\min & U_{1}\left(f\left(T, \xi_{n}\right)\right) \\
\text { s.t. } & \text { constraints (4)-(9). }
\end{aligned}
$$

It is clear that, the MV model (19) is quite complicated because it involves a variety of constraints and contains many variables. Ibarra-Rojas and Rios-Solis [31] have shown that the train timetabling problem belongs to the NP-hard class problem set because of the large number of variables and constraints. In order to obtain a satisfactory last-train timetable within an acceptable time, we design a TS algorithm with specifically designed operators to solve the MV model (19) in this paper.

\section{Tabu Search Algorithm}

For train timetabling problems, researchers usually use a very large number of different techniques, including exact and approximation algorithms. Among these solution approaches, the TS algorithm developed by Glover [32] has been very successful in finding close to optimal solutions for engineering problems [33-37]. The TS algorithm starts searching with an initial solution, based on which a set of feasible solutions is constructed as the neighborhood. Then the procedure will select the best local candidate among the neighborhood (although it has worse fitness than the current best) as the next current solution in order to escape the local optimal. The history record, that is, the tabu list, is modified after it. The process is repeated until the user-specified stopping criteria are satisfied. Finally, the best solution during the search process will be returned.
First, we introduce some notations. Let Initial_s be an initial solution and formed as an array composed by two vectors representing the departure time of last trains from origin stations and the segment running times, as shown in Figure 3. In this paper, we input original values of the two vectors as an initial solution. Given a solution Present_s, we define the set $N$ (Present_s) to be the set of neighboring solutions. To obtain the neighboring solutions, we applied the procedure illustrated in Figure 4.

Step 1 (selection). Randomly select $k$ positions in an array to make changes based on Present_s.

Step 2 (change). Produce values at the $k$ picked positions based on the Solution Generation Algorithm [38], while meeting constraints (8) and (9).

Step 3 (substitute). A new solution is generated by replacing the corresponding values with the newly generated ones in Present_s.

Based on the aforementioned analyses, the procedure of this heuristic algorithm is summarized by Algorithm 1 .

\section{Case Study}

In this section, we verify the effectiveness of the TS algorithm and the application of the MV model using real cases from the Beijing subway system. The proposed TS algorithm has been coded in MATLAB 2013a. All numerical tests are carried out on a Windows Server 2008 R2 Enterprise workstation with Intel ${ }^{\circledR}$ Xeon ${ }^{\circledR}$ CPU E5-2640v3 @ $2.60 \mathrm{GHz}$ and $64 \mathrm{G}$ RAM to improve the solving efficiency.

Next, we perform this case study on the practical Beijing subway network. Since this paper focuses only on transfer between different lines, we simplify the original Beijing 


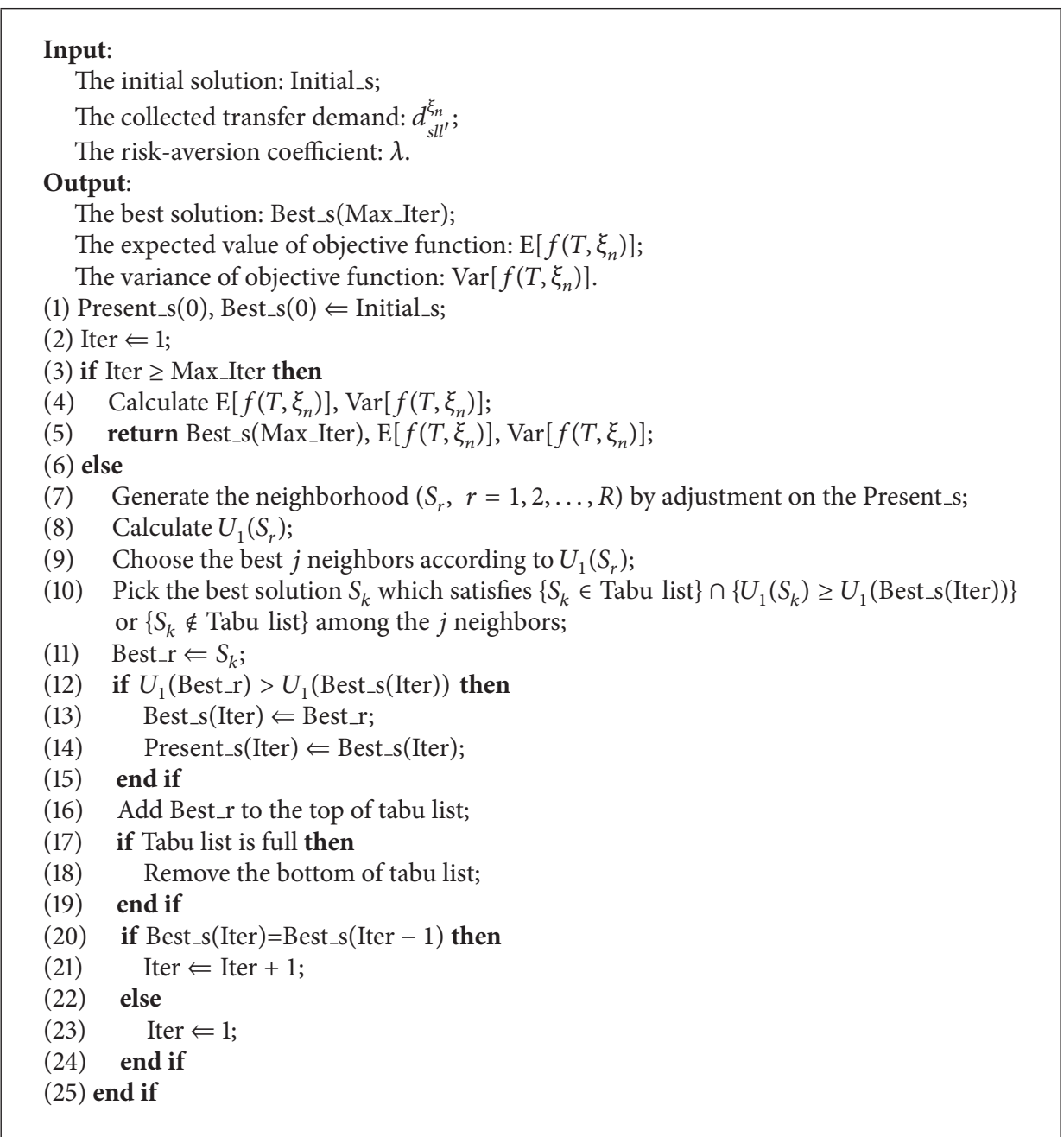

Algorithm 1: TS algorithm procedure.

Subway Map for computational convenience. In detail, we give a simplified structure of the considered Beijing subway network in Figure 5, which includes 12 lines, 45 transfer stations, and 81 segments. Note that T1, T2,.., T24 symbol different last trains with directions suggested by the arrows next to them.

5.1. Data Collection. Before conducting the experiments, we need to precollect some basic data in the solution process. More specifically, we adopt ten-day transfer demands (i.e., 10 samples) during the last-train period (between 22:00 and 24:00 daily) as the experimental data. In addition, we set the probability of each sample $p_{\xi_{n}}$ as 0.1 , which is described in Section 3.2.1. Tables 1 and 2 demonstrate the present timetable of last trains in Beijing subway. Note that 22:00 is set as the zero point for simplicity in the process and 50 means the 22:50 in 24 hour clock, to take an example.

5.2. Computational Results. After implementing the proposed model and solution approach, we examine the effect of varying risk-aversion coefficients $\lambda$ on the expected value of $f\left(T, \xi_{n}\right)$ and its standard deviation, as summarized in
Figures 6 and 7, respectively. As anticipated, the expected value of objective function increases as the risk-aversion coefficient increases, while the standard deviation of objective function takes an opposite tendency with respective to riskaversion coefficient. Furthermore, it is obvious that there are unique decisions for each level of risk-aversion coefficient.

Figure 8 demonstrates the three-dimensional characteristics of the relationship among $\mu, \sigma$, and $\lambda$. This curve contains all the different preferences of decision-makers. In other words, there will be a risk-aversion coefficient for every preference of the DM $(\mu, \sigma)$ and each risk-aversion coefficient corresponds to a certain preference and then the curve consists of all the risk-aversion DMs' preferences. The value of risk-aversion coefficient is chosen based on the DM's preferences and his/her risk attitude, and it may be different for each DM. Therefore, the DM should be able to select a risk-aversion coefficient on the curve based on their preference to obtain a reliable decision for implementation.

5.3. Comparison Results. To further validate the advantages of the proposed MV model, more computational results were obtained to solve a similar problem with expected utility 
TABLE 1: Initial starting time of last trains in Beijing subway network (unit: min).

\begin{tabular}{|c|c|c|c|c|c|c|c|c|c|c|c|c|c|c|c|c|c|c|c|c|c|c|c|c|}
\hline Train & $\mathrm{T} 1$ & T2 & T3 & $\mathrm{T} 4$ & T5 & T6 & T7 & T8 & T9 & T10 & T11 & $\mathrm{T} 12$ & $\mathrm{~T} 13$ & $\mathrm{~T} 14$ & T15 & T16 & T17 & T18 & T19 & T20 & $\mathrm{T} 21$ & T22 & T23 & $\mathrm{T} 24$ \\
\hline$t_{o l}^{D}$ & 55 & 42 & 60 & 42 & 38 & 20 & 71 & 48 & 79 & 49 & 75 & 25 & 60 & 5 & 79 & 40 & 41 & 32 & 42 & 42 & 40 & 30 & 75 & 11 \\
\hline
\end{tabular}

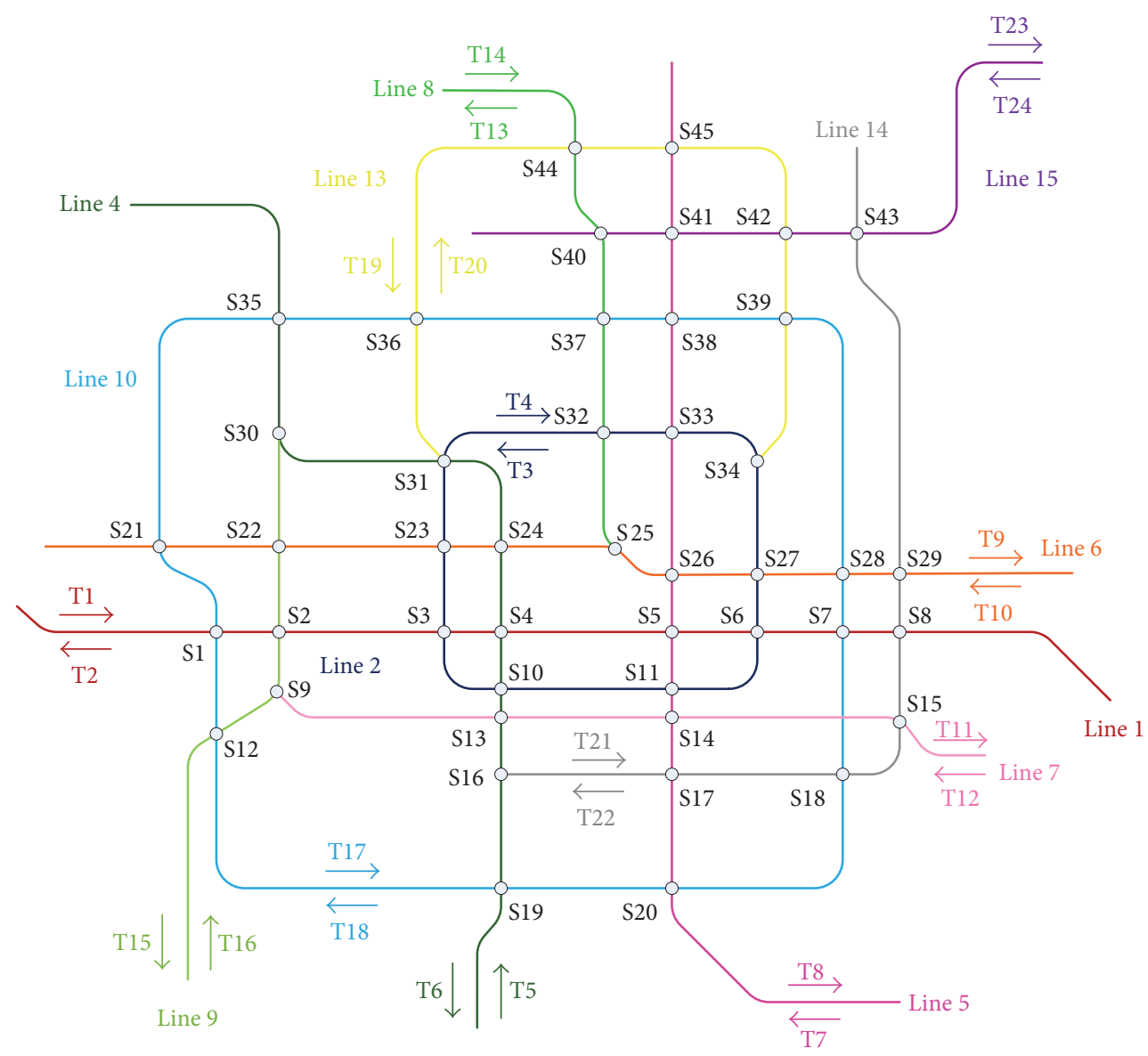

FIGURE 5: Simplified Beijing Subway Map.

criteria. When $\lambda=0$, the MV model is degenerated to expectation utility model. Table 3 summarizes the comparison of solution results on different risk-aversion coefficient $\lambda$. It can be observed that the variance of objective function in expectation utility model is higher than that in MV model in terms of optimal solutions. This result suggests that the MV model obtains better results compared to its counterpart with expected utility criteria in a risky situation. Furthermore, the MV model can also provide comprehensive strategies to generate the last-train timetables according to the DM' preferences.

\section{Conclusions}

Focusing on generating timetables for last trains, this paper proposed a modeling framework in subway systems with uncertainty. Specifically, the sample-based transfer demands were adopted to represent the randomness in the subway network. Considering the data structure, the mean-variance utility based model for last-train timetabling problems was formulated to fully capture the risk preference under uncertainty. Then, the mean-variance risk measure was added to the proposed model. One of the main problems associated with this measure is heterogeneity between the variance and the expected value in the objective function. In order to eliminate the effect of this heterogeneity, a normalization method was employed, which is of huge assistance in getting reasonable results. To solve the models more efficiently, a TS based algorithmic framework was proposed that can obtain a good solution in an acceptable computational time. Finally, a practical case study of the Beijing subway system demonstrated the effectiveness of the proposed model and optimization method.

Future research can focus on the following several aspects. (1) The improvements to the algorithm efficiency, designing hybrid algorithms, or developing an adaptive method can be considered in the further study. (2) The influence of feeder train delay needs to be studied in advance. (3) Except for the mean-variation criterion when coping with risk-aversion preferences, we should carry out some other methods and investigate which can better signify risk levels in our future research. 
TABLE 2: Transfer-station arriving time of last trains and successful transfers in Beijing subway network.

\begin{tabular}{|c|c|c|c|c|c|c|c|c|c|}
\hline Station & Train & Arr. time & Train & Arr. time & Train & Arr. time & Train & Arr. time & Successful transfers \\
\hline S1 & $\mathrm{T} 1$ & 75 & $\mathrm{~T} 2$ & 110 & T17 & 49 & T18 & 122 & 181 \\
\hline S2 & $\mathrm{T} 1$ & 78 & $\mathrm{~T} 2$ & 107 & $\mathrm{~T} 15$ & 87 & $\mathrm{~T} 16$ & 63 & 188 \\
\hline S3 & $\mathrm{T} 1$ & 85 & $\mathrm{~T} 2$ & 101 & $\mathrm{~T} 3$ & 67 & $\mathrm{~T} 4$ & 79 & 153 \\
\hline S4 & $\mathrm{T} 1$ & 87 & $\mathrm{~T} 2$ & 98 & $\mathrm{~T} 5$ & 85 & T6 & 55 & 222 \\
\hline S5 & $\mathrm{T} 1$ & 95 & $\mathrm{~T} 2$ & 90 & $\mathrm{~T} 7$ & 84 & Т8 & 84 & 184 \\
\hline S6 & $\mathrm{T} 1$ & 98 & $\mathrm{~T} 2$ & 88 & T3 & 83 & $\mathrm{~T} 4$ & 63 & 221 \\
\hline S7 & $\mathrm{T} 1$ & 102 & $\mathrm{~T} 2$ & 83 & $\mathrm{~T} 17$ & 99 & $\mathrm{~T} 18$ & 73 & 206 \\
\hline S8 & $\mathrm{T} 1$ & 105 & $\mathrm{~T} 2$ & 81 & $\mathrm{~T} 21$ & 64 & $\mathrm{~T} 22$ & 57 & 192 \\
\hline S9 & T11 & 75 & $\mathrm{~T} 12$ & 67 & $\mathrm{~T} 15$ & 90 & T16 & 60 & 251 \\
\hline S10 & $\mathrm{T} 3$ & 71 & $\mathrm{~T} 4$ & 74 & $\mathrm{~T} 5$ & 83 & T6 & 57 & 207 \\
\hline S11 & $\mathrm{T} 3$ & 78 & $\mathrm{~T} 4$ & 68 & $\mathrm{~T} 7$ & 82 & $\mathrm{~T} 8$ & 86 & 197 \\
\hline S12 & $\mathrm{T} 15$ & 95 & T16 & 54 & $\mathrm{~T} 17$ & 55 & $\mathrm{~T} 18$ & 116 & 196 \\
\hline S13 & T5 & 80 & T6 & 58 & T11 & 84 & $\mathrm{~T} 12$ & 58 & 296 \\
\hline S14 & $\mathrm{T} 7$ & 80 & $\mathrm{~T} 8$ & 88 & T11 & 93 & $\mathrm{~T} 12$ & 50 & 226 \\
\hline S15 & T11 & 101 & $\mathrm{~T} 12$ & 41 & $\mathrm{~T} 21$ & 61 & $\mathrm{~T} 22$ & 60 & 182 \\
\hline S16 & $\mathrm{T} 5$ & 75 & T6 & 63 & $\mathrm{~T} 21$ & 40 & $\mathrm{~T} 22$ & 81 & 200 \\
\hline S17 & $\mathrm{T} 7$ & 75 & $\mathrm{~T} 8$ & 93 & $\mathrm{~T} 21$ & 48 & $\mathrm{~T} 22$ & 74 & 200 \\
\hline S18 & T17 & 90 & T18 & 81 & $\mathrm{~T} 21$ & 53 & $\mathrm{~T} 22$ & 68 & 206 \\
\hline S19 & $\mathrm{T} 5$ & 71 & T6 & 68 & $\mathrm{~T} 17$ & 74 & T18 & 97 & 150 \\
\hline S20 & $\mathrm{T} 7$ & 71 & T8 & 97 & T17 & 82 & T18 & 89 & 216 \\
\hline S21 & T9 & 81 & $\mathrm{~T} 10$ & 116 & $\mathrm{~T} 17$ & 44 & $\mathrm{~T} 18$ & 127 & 171 \\
\hline S22 & $\mathrm{T} 9$ & 86 & $\mathrm{~T} 10$ & 111 & $\mathrm{~T} 15$ & 81 & T16 & 68 & 219 \\
\hline S23 & T3 & 62 & $\mathrm{~T} 4$ & 84 & Т9 & 91 & $\mathrm{~T} 10$ & 106 & 203 \\
\hline S24 & $\mathrm{T} 5$ & 90 & T6 & 48 & T9 & 94 & $\mathrm{~T} 10$ & 104 & 228 \\
\hline S25 & T9 & 99 & $\mathrm{~T} 10$ & 99 & $\mathrm{~T} 13$ & 60 & $\mathrm{~T} 14$ & 51 & 202 \\
\hline S26 & $\mathrm{T} 7$ & 88 & $\mathrm{~T} 8$ & 80 & T9 & 103 & $\mathrm{~T} 10$ & 95 & 223 \\
\hline S27 & $\mathrm{T} 3$ & 86 & $\mathrm{~T} 4$ & 60 & T9 & 105 & $\mathrm{~T} 10$ & 92 & 181 \\
\hline S28 & T9 & 110 & $\mathrm{~T} 10$ & 87 & $\mathrm{~T} 17$ & 103 & T18 & 69 & 172 \\
\hline S29 & $\mathrm{T} 9$ & 113 & $\mathrm{~T} 10$ & 85 & $\mathrm{~T} 21$ & 67 & $\mathrm{~T} 22$ & 54 & 202 \\
\hline S30 & T5 & 99 & T6 & 39 & $\mathrm{~T} 15$ & 79 & T16 & 70 & 192 \\
\hline \multirow{2}{*}{ S31 } & $\mathrm{T} 3$ & 60 & $\mathrm{~T} 4$ & 42 & $\mathrm{~T} 5$ & 95 & T6 & 45 & \multirow{2}{*}{656} \\
\hline & T19 & 98 & $\mathrm{~T} 20$ & 42 & - & - & - & - & \\
\hline S32 & T3 & 98 & $\mathrm{~T} 4$ & 48 & $\mathrm{~T} 13$ & 64 & $\mathrm{~T} 14$ & 45 & 193 \\
\hline S33 & $\mathrm{T} 3$ & 94 & $\mathrm{~T} 4$ & 52 & $\mathrm{~T} 7$ & 94 & $\mathrm{~T} 8$ & 74 & 295 \\
\hline S34 & $\mathrm{T} 3$ & 90 & $\mathrm{~T} 4$ & 56 & T19 & 42 & T20 & 97 & 201 \\
\hline S35 & $\mathrm{T} 5$ & 107 & T6 & 33 & $\mathrm{~T} 17$ & 136 & T18 & 36 & 206 \\
\hline S36 & $\mathrm{T} 17$ & 132 & $\mathrm{~T} 18$ & 40 & T19 & 92 & $\mathrm{~T} 20$ & 49 & 181 \\
\hline S37 & $\mathrm{T} 13$ & 70 & $\mathrm{~T} 14$ & 39 & $\mathrm{~T} 17$ & 123 & $\mathrm{~T} 18$ & 48 & 176 \\
\hline S38 & T7 & 101 & T8 & 68 & $\mathrm{~T} 17$ & 119 & $\mathrm{~T} 18$ & 53 & 219 \\
\hline S39 & T17 & 116 & T18 & 56 & T19 & 49 & T20 & 90 & 186 \\
\hline S40 & $\mathrm{T} 13$ & 75 & $\mathrm{~T} 14$ & 34 & $\mathrm{~T} 23$ & 82 & $\mathrm{~T} 24$ & 62 & 181 \\
\hline S41 & T7 & 105 & $\mathrm{~T} 8$ & 63 & $\mathrm{~T} 23$ & 87 & $\mathrm{~T} 24$ & 57 & 179 \\
\hline S42 & T19 & 52 & T20 & 87 & $\mathrm{~T} 23$ & 92 & $\mathrm{~T} 24$ & 51 & 191 \\
\hline S43 & $\mathrm{T} 21$ & 85 & $\mathrm{~T} 22$ & 36 & $\mathrm{~T} 23$ & 96 & $\mathrm{~T} 24$ & 48 & 179 \\
\hline S44 & $\mathrm{T} 13$ & 92 & $\mathrm{~T} 14$ & 17 & $\mathrm{~T} 19$ & 68 & $\mathrm{~T} 20$ & 71 & 216 \\
\hline S45 & $\mathrm{T} 7$ & 114 & $\mathrm{~T} 8$ & 54 & $\mathrm{~T} 19$ & 63 & T20 & 77 & 195 \\
\hline
\end{tabular}


TABLE 3: Comparison of solution results.

\begin{tabular}{lccccc}
\hline$\lambda$ & Total running time & Successful transfers & Expected value & Variance & Objective value \\
\hline 0 & 1115.7 & 10425 & -201.1680 & 471590 & 0.0015 \\
0.2 & 1128.6 & 10166 & -193.4117 & 422460 & 0.2821 \\
0.4 & 1116.8 & 10170 & -192.1094 & 416190 & 0.3929 \\
0.6 & 1101.9 & 9872.4 & -185.9823 & 358560 & 0.4870 \\
0.8 & 1064.3 & 9574.7 & -180.8096 & 318390 & 0.5056 \\
1.0 & 1063.7 & 9528.5 & -179.4860 & 305110 & 0.5141 \\
\hline
\end{tabular}

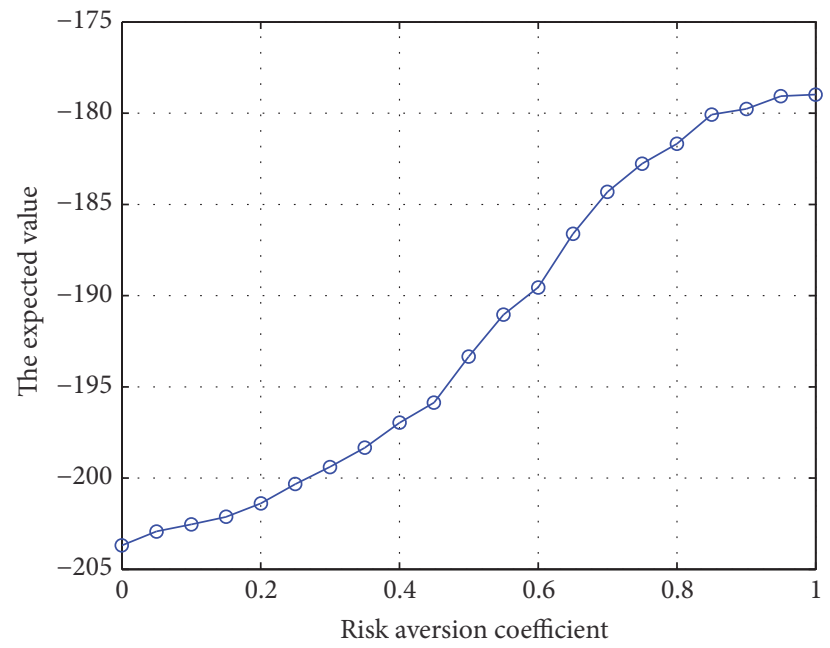

FIgURE 6: The expected value for different risk-aversion coefficient $\lambda$.

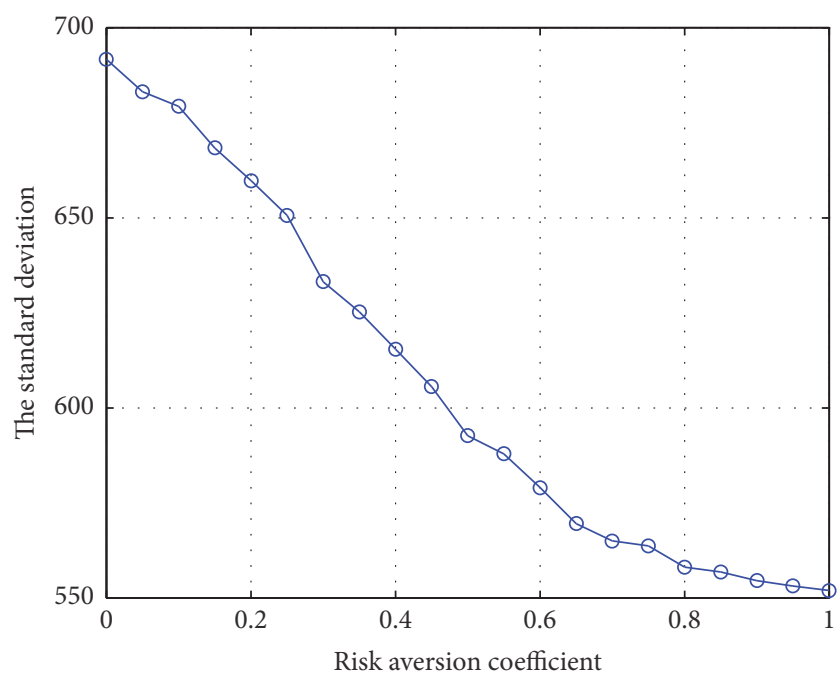

FIGURE 7: The standard variation for different risk-aversion coefficient $\lambda$.

\section{Conflicts of Interest}

The authors declare that there are no conflicts of interest regarding the publication of this paper.

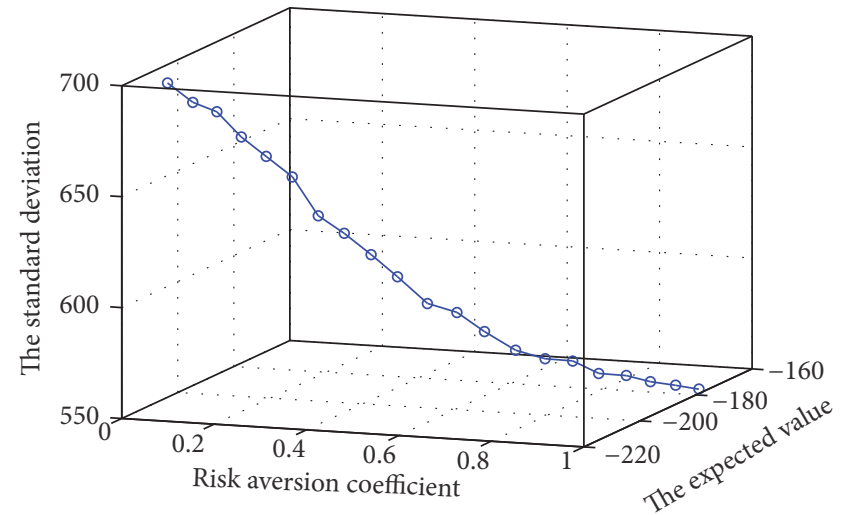

Figure 8: The three-dimensional demonstration of relationships among $\mu, \sigma$, and $\lambda$.

\section{Acknowledgments}

This work was supported by the National Natural Science Foundation of China (nos. 71422002, 71621001, 71631007, and 71701012), the Research Foundation of the State Key Laboratory of Rail Traffic Control and Safety, Beijing Jiaotong University (no. RCS2017ZZ01), and the Fundamental Research Funds of Beijing Jiaotong University (no. 2015RC075).

\section{References}

[1] J. Törnquist Krasemann, "Design of an effective algorithm for fast response to the re-scheduling of railway traffic during disturbances," Transportation Research Part C: Emerging Technologies, vol. 20, no. 1, pp. 62-78, 2012.

[2] L. Peeters and L. Kroon, "A Cycle Based Optimization Model for the Cyclic Railway Timetabling Problem," in ComputerAided Scheduling of Public Transport, vol. 505 of Lecture Notes in Economics and Mathematical Systems, pp. 275-296, Springer Berlin Heidelberg, Berlin, Heidelberg, 2001.

[3] T. Albrecht and S. Oettich, "A new integrated approach to dynamic schedule synchronization and energy-saving train control," in Computers in Railways VIII, pp. 847-856, WIT Press, Southampton, UK, 2002.

[4] C. Liebchen, "The first optimized railway timetable in practice," Transportation Science, vol. 42, no. 4, pp. 420-435, 2008.

[5] P. Stanojević, M. Marić, J. Kratica, N. Bojović, and M. Milenković, "Mathematical optimization for the train timetabling problem," Mathematica Balkanica New Series, vol. 24, no. 3, pp. 303-312, 2010. 
[6] J. Yin, L. Yang, T. Tang, Z. Gao, and B. Ran, "Dynamic passenger demand oriented metro train scheduling with energyefficiency and waiting time minimization: Mixed-integer linear programming approaches," Transportation Research Part B: Methodological, vol. 97, pp. 182-213, 2017.

[7] Y. Gao, L. Kroon, M. Schmidt, and L. Yang, "Rescheduling a metro line in an over-crowded situation after disruptions," Transportation Research Part B: Methodological, vol. 93, pp. 425449, 2016.

[8] Y. Huang, L. Yang, T. Tang, Z. Gao, and F. Cao, "Joint train scheduling optimization with service quality and energy efficiency in urban rail transit networks," Energy, vol. 138, pp. 11241147, 2017.

[9] W. Zhou, L. Deng, M. Xie, and X. Yang, "Coordination optimization of the first and last trains' departure time on urban rail transit network," Advances in Mechanical Engineering, vol. 5, no. 3, pp. 848292-848292, 2013.

[10] L. Kang, J. Wu, H. Sun, X. Zhu, and B. Wang, "A practical model for last train rescheduling with train delay in urban railway transit networks," Omega, vol. 50, pp. 29-42, 2015.

[11] L. Kang, J. Wu, H. Sun, X. Zhu, and Z. Gao, "A case study on the coordination of last trains for the Beijing subway network," Transportation Research Part B: Methodological, vol. 72, pp. 112127, 2015.

[12] W. Li, R. Xu, Q. Luo, and S. Jones, "Coordination of last train transfers using automated fare collection (AFC) system data," Journal of Advanced Transportation, vol. 50, no. 8, pp. 22092225, 2016.

[13] L. X. Yang, K. P. Li, and Z. Y. Gao, “Train timetable problem on a single-line railway with fuzzy passenger demand," IEEE Transactions on Fuzzy Systems, vol. 17, no. 3, pp. 617-629, 2009.

[14] E. Barrena, D. Canca, L. C. Coelho, and G. Laporte, "Singleline rail rapid transit timetabling under dynamic passenger demand," Transportation Research Part B: Methodological, vol. 70, pp. 134-150, 2014.

[15] J. Yin, T. Tang, L. Yang, Z. Gao, and B. Ran, "Energy-efficient metro train rescheduling with uncertain time-variant passenger demands: An approximate dynamic programming approach," Transportation Research Part B: Methodological, vol. 91, pp. 178210, 2016.

[16] J. Yin, D. Chen, L. Yang, T. Tang, and B. Ran, "Efficient RealTime Train Operation Algorithms with Uncertain Passenger Demands," IEEE Transactions on Intelligent Transportation Systems, vol. 17, no. 9, pp. 1-13, 2016.

[17] H.-H. Choi, H.-N. Cho, and J. W. Seo, "Risk assessment methodology for underground construction projects," Journal of Construction Engineering and Management, vol. 130, no. 2, pp. 258-272, 2004.

[18] D. A. Hensher, Z. Li, and J. M. Rose, "Accommodating risk in the valuation of expected travel time savings," Journal of Advanced Transportation, vol. 47, no. 2, pp. 206-224, 2013.

[19] M. Woodward, Z. Kapelan, and B. Gouldby, "Adaptive flood risk management under climate change uncertainty using real options and optimization," Risk Analysis, vol. 34, no. 1, pp. 7592, 2014.

[20] E. Zio, "Challenges in the vulnerability and risk analysis of critical infrastructures," Reliability Engineering and System Safety, vol. 152, pp. 137-150, 2016.

[21] X. Liu, M. Rapik Saat, and C. P. L. Barkan, "Freight-train derailment rates for railroad safety and risk analysis," Accident Analysis and Prevention, vol. 98, pp. 1-9, 2017.
[22] H. Markowitz, "Portfolio selection," The Journal of Finance, vol. 7, no. 1, pp. 77-91, 1952.

[23] T.-M. Choi, D. Li, H. Yan, and C.-H. Chiu, "Channel coordination in supply chains with agents having mean-variance objectives," Omega, vol. 36, no. 4, pp. 565-576, 2008.

[24] C. Fu, A. Lari-Lavassani, and X. Li, "Dynamic mean-variance portfolio selection with borrowing constraint," European Journal of Operational Research, vol. 200, no. 1, pp. 312-319, 2010.

[25] J. J. Wu, L. J. Kang, H. J. Sun, and X. L. Jia, “Track allocation optimization in railway station: mean-variance model and case study," Journal of Transportation Engineering, vol. 139, no. 5, pp. 540-547, 2013.

[26] T. Ahmadi, H. Karimi, H. Davoudpour, and S. A. Hosseinijou, "A robust decision-making approach for $\mathrm{p}$-hub median location problems based on two-stage stochastic programming and mean-variance theory: a real case study," International Journal of Advanced Manufacturing Technology, vol. 77, no. 9-12, pp. 19431953, 2015.

[27] H. Li, H. Tu, and D. A. Hensher, "Integrating the mean-variance and scheduling approaches to allow for schedule delay and trip time variability under uncertainty," Transportation Research Part A: Policy and Practice, vol. 89, pp. 151-163, 2016.

[28] R. C. W. Wong, T. W. Y. Yuen, K. W. Fung, and J. M. Y. Leung, "Optimizing timetable synchronization for rail mass transit," Transportation Science, vol. 42, no. 1, pp. 57-69, 2008.

[29] J. R. Birge and F. Louveaux, Introduction to Stochastic Programming, Springer, 1997.

[30] A. J. Kleywegt, A. Shapiro, and T. Homem-de-Mello, "The sample average approximation method for stochastic discrete optimization," SIAM Journal on Optimization, vol. 12, no. 2, pp. 479-502, 2002.

[31] O. J. Ibarra-Rojas and Y. A. Rios-Solis, "Synchronization of bus timetabling," Transportation Research Part B: Methodological, vol. 46, no. 5, pp. 599-614, 2012.

[32] F. Glover, "Future paths for integer programming and links to artificial intelligence," Computers \& Operations Research, vol. 13, no. 5, pp. 533-549, 1986.

[33] F. Glover, T. Ye, A. P. Punnen, and G. Kochenberger, "Integrating tabu search and VLSN search to develop enhanced algorithms: a case study using bipartite boolean quadratic programs," European Journal of Operational Research, vol. 241, no. 3, pp. 697-707, 2015.

[34] Z. Xue, C. Zhang, W.-H. Lin, L. Miao, and P. Yang, "A tabu search heuristic for the local container drayage problem under a new operation mode," Transportation Research Part E: Logistics and Transportation Review, vol. 62, pp. 136-150, 2014.

[35] J. Paquette, J.-F. Cordeau, G. Laporte, and M. M. B. Pascoal, "Combining multicriteria analysis and tabu search for dial-aride problems," Transportation Research Part B: Methodological, vol. 52, pp. 1-16, 2013.

[36] F. Ruisanchez, L. Dell'Olio, and A. Ibeas, "Design of a tabu search algorithm for assigning optimal bus sizes and frequencies in urban transport services," Journal of Advanced Transportation, vol. 46, no. 4, pp. 366-377, 2012.

[37] O. Shahvari and R. Logendran, "An enhanced tabu search algorithm to minimize a bi-criteria objective in batching and scheduling problems on unrelated-parallel machines with desired lower bounds on batch sizes," Computers \& Operations Research, vol. 77, pp. 154-176, 2017. 
[38] L. Kang and X. Zhu, "A simulated annealing algorithm for first train transfer problem in urban railway networks," Applied Mathematical Modelling. Simulation and Computation for Engineering and Environmental Systems, vol. 40, no. 1, pp. 419-435, 2016. 


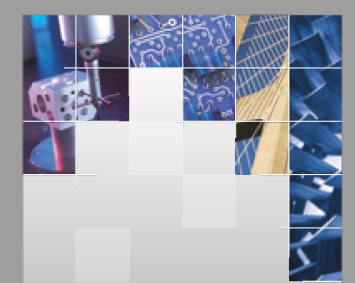

\section{Enfincering}
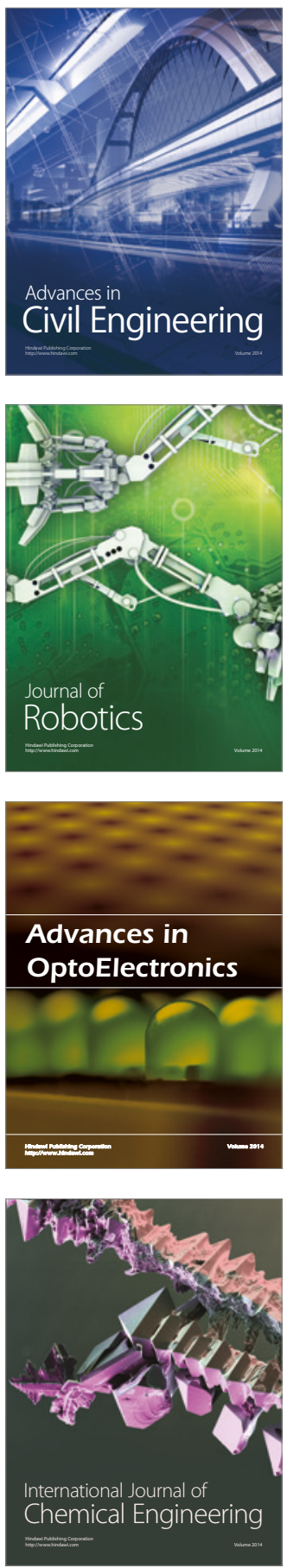

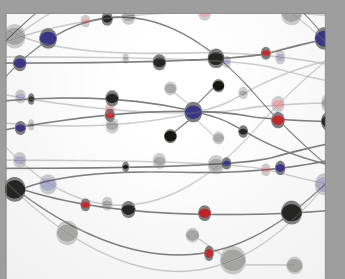

The Scientific World Journal

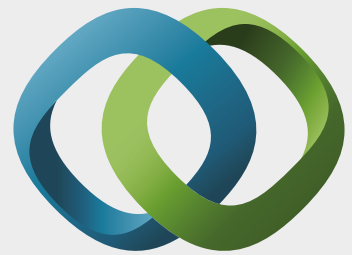

\section{Hindawi}

Submit your manuscripts at

https://www.hindawi.com
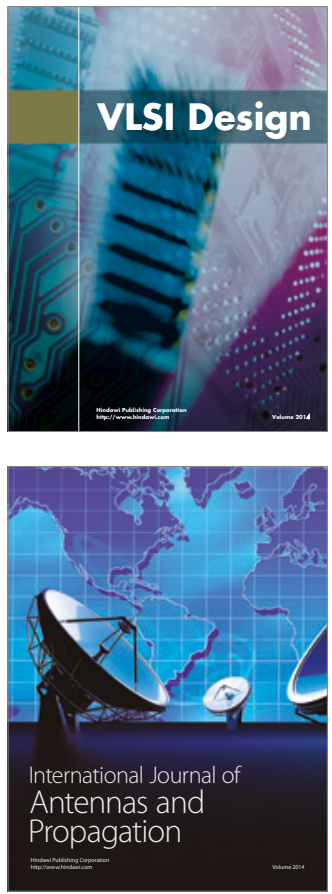

\section{Rotating}

Machinery
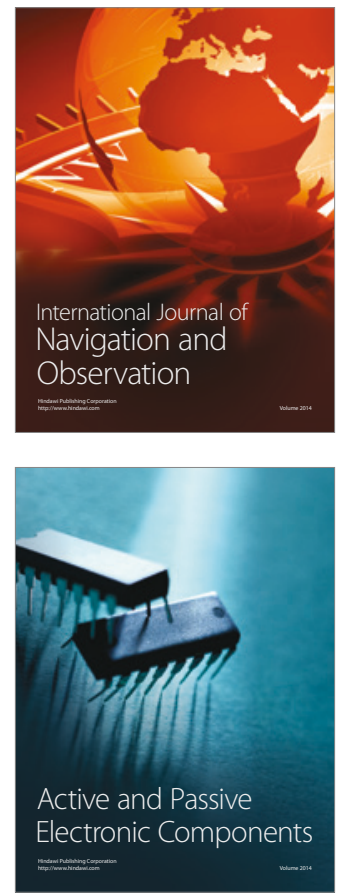
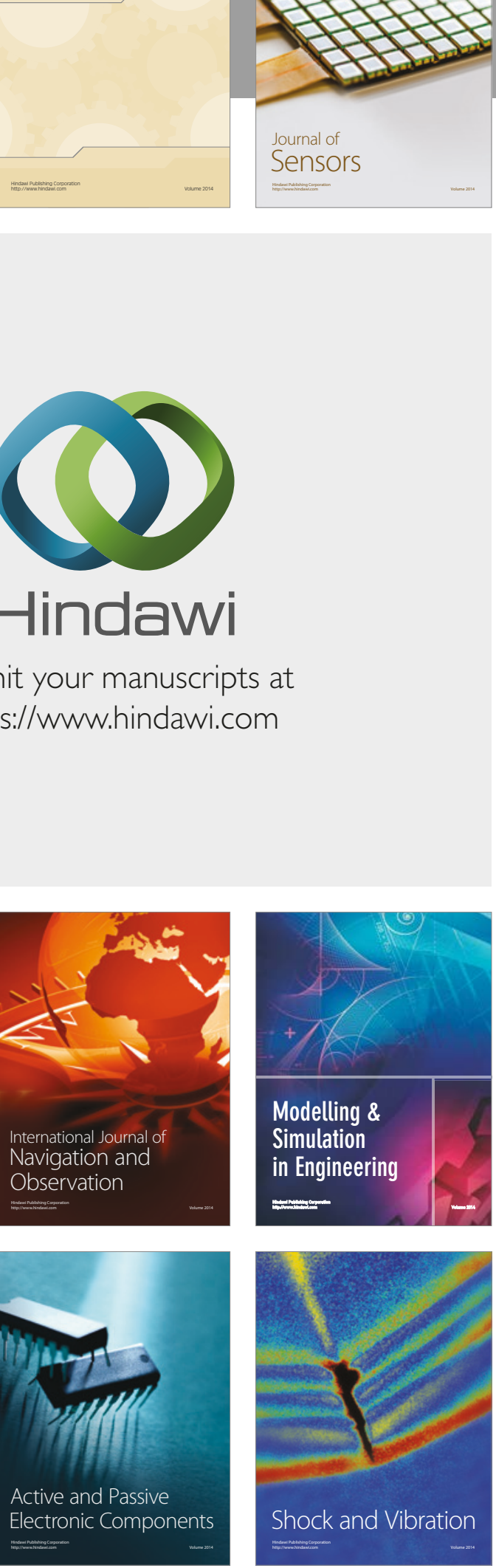
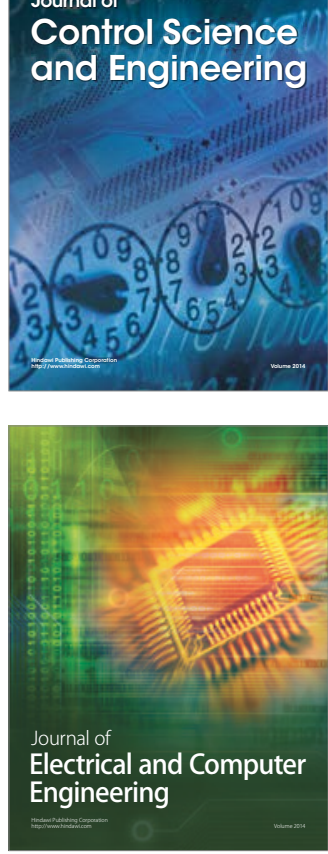

Distributed

Journal of

Control Science

and Engineering
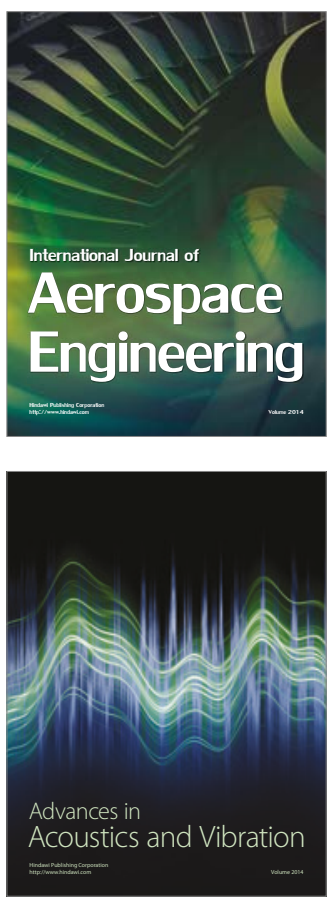

Sensor Networks 